\title{
Terahertz time-domain spectroscopy of crystalline and aqueous systems
}

\author{
Jepsen, Peter Uhd; Møller, Uffe; Eichhorn, Finn; Merbold, Hannes; Folkenberg, J.R.; Clark, S.J.
}

Published in:

Conference on Lasers and Electro-Optics, 2007. CLEO 2007.

Link to article, DOI:

10.1109/CLEO.2007.4452447

Publication date:

2007

Document Version

Publisher's PDF, also known as Version of record

Link back to DTU Orbit

Citation (APA):

Jepsen, P. U., Møller, U., Eichhorn, F., Merbold, H., Folkenberg, J. R., \& Clark, S. J. (2007). Terahertz timedomain spectroscopy of crystalline and aqueous systems. In Conference on Lasers and Electro-Optics, 2007. CLEO 2007. (pp. 1-2). IEEE. https://doi.org/10.1109/CLEO.2007.4452447

\section{General rights}

Copyright and moral rights for the publications made accessible in the public portal are retained by the authors and/or other copyright owners and it is a condition of accessing publications that users recognise and abide by the legal requirements associated with these rights.

- Users may download and print one copy of any publication from the public portal for the purpose of private study or research.

- You may not further distribute the material or use it for any profit-making activity or commercial gain

- You may freely distribute the URL identifying the publication in the public portal 


\title{
Terahertz time-domain spectroscopy of crystalline and aqueous systems
}

\author{
Peter Uhd Jepsen ${ }^{1}$, Uffe Møller ${ }^{1}$, Finn Eichhorn ${ }^{1}$, Hannes Merbold ${ }^{2}$, Jacob Riis Folkenberg ${ }^{3}$, and Stewart J. \\ Clark $^{4}$ \\ ${ }^{1}$ COM $\bullet D T U$, Technical University of Denmark, DK-2800 Kongens Lyngby, Denmark \\ ${ }^{2}$ Institute of Mathematics and Physics, University of Freiburg, D-79104 Freiburg, Germany \\ ${ }^{3}$ Foss A/S, Slangerupgade 69, DK-3400 Hillerød, Denmark \\ ${ }^{4}$ Department of Physics, University of Durham, Durham DH1 3LE, United Kingdom, \\ Authoremail: uffe@com.dtu.dk, jepsen@com.dtu.dk
}

\begin{abstract}
We use ab-initio Density-Functional Perturbation Theory together with $\mathrm{THz}$ spectroscopy for precise prediction and assignment of vibrational modes in molecular crystals. We show that THz spectroscopy is useful for analysis of liquids and food products.

(C)2007 Optical Society of America

OCIS Codes: (300.6270) Spectroscopy, far-infrared (170.1580) Chemometrics
\end{abstract}

\section{Introduction}

In the recent years there has been a tremendous activity in the field of basic and applied THz frequency research. A sizable fraction of this effort has been focused on the exploitation of the fact that most organic molecules in the solid state have a rich and distinct dielectric spectrum in the THz region 0.3-5 THz. It has turned out that the vibrational modes found in this particular region of the electromagnetic spectrum are highly characteristic not only for the molecule, but also for its environment. The space group symmetry of the unit cell as well as the content of cocrystallized solvent molecules (e.g. water) has been shown to be the main factors forming the $\mathrm{THz}$ vibrational spectrum of the solid.

The richly structured dielectric spectra often observed in poly- and single-crystal materials, including powders, are due to combinations of phonon- and intramolecular modes of the crystallites or single-crystals. On the other hand, in amorphous condensed-phase systems the existence of spectrally localized features is extremely rare because of the strong coupling between the random environment and intramolecular modes [1]. This study showed that the long-range order of the environment of the molecules is one of the dominating factors in the shaping of the dielectric spectrum of the molecules.

Here we present a generally applicable ab-initio simulation method that is capable of predicting the position and intensity, as well as identifying the normal modes of vibrational spectra in the THz region. The applicability of the method is demonstrated with results of the simulation of vibrational modes of the hydrogen-bonded molecular crystal sucrose. The experimentally determined absorption spectrum of polycrystalline sucrose has been reported earlier [1].

In line with the rising demands on increased food quality and safety there is a need of new and fast measuring methods for rapid and objective determination of the state and quality of the food product on its way from the producer to the consumer. However, the development of new optical measuring methods for food control still implies fundamental studies of the interaction between light and matter and it is therefore not only an important issue for the food industry but also interesting from a general scientific point of view.

The spectral regions of NIR and mid-IR typically give intramolecular information such as the presence of different characteristic bonds, e.g. C-O, C-C, C-H and C-N, thus indicating the presence of certain molecules or groups of molecules in the sample. However, these spectral ranges give poor intermolecular information, e.g. the alignment of molecules, hydrogen bonds to surrounding molecules and whether the molecules are a part of an amorphous or crystalline structure. This information is to be found at longer wavelengths, namely in the terahertz region.

In spite of the lack of spectral features in the $\mathrm{THz}$ range of the dielectric function of amorphous materials and aqueous solutions, THz spectroscopy turns out to be very useful for identification also of ingredients in solution, and also to determine their concentration with high accuracy. We will show how $\mathrm{THz}$ reflection spectroscopy can be used to determine the alcohol concentration with high accuracy in a wide range of beverages such as beer, wine and spirits, independent of the presence of microparticles, carbon dioxide, and other ingredients. 


\section{CFS1.pdf}

\section{Selected results}

To accurately predict the low frequency modes, which are of strong intermolecular nature, the full periodic structure of the crystal must be considered. The calculations are based on the plane-wave density functional method within the generalised gradient approximation as implemented in the Castep code [3]. In Fig. 1 (left panel) we show the result of a simulation of the vibrational spectrum of the sucrose crystal. The vertical bars correspond to the calculated vibrational frequencies, and the solid curve is the experimentally determined absorption spectrum of sucrose, measured at a temperature of $10 \mathrm{~K}$.
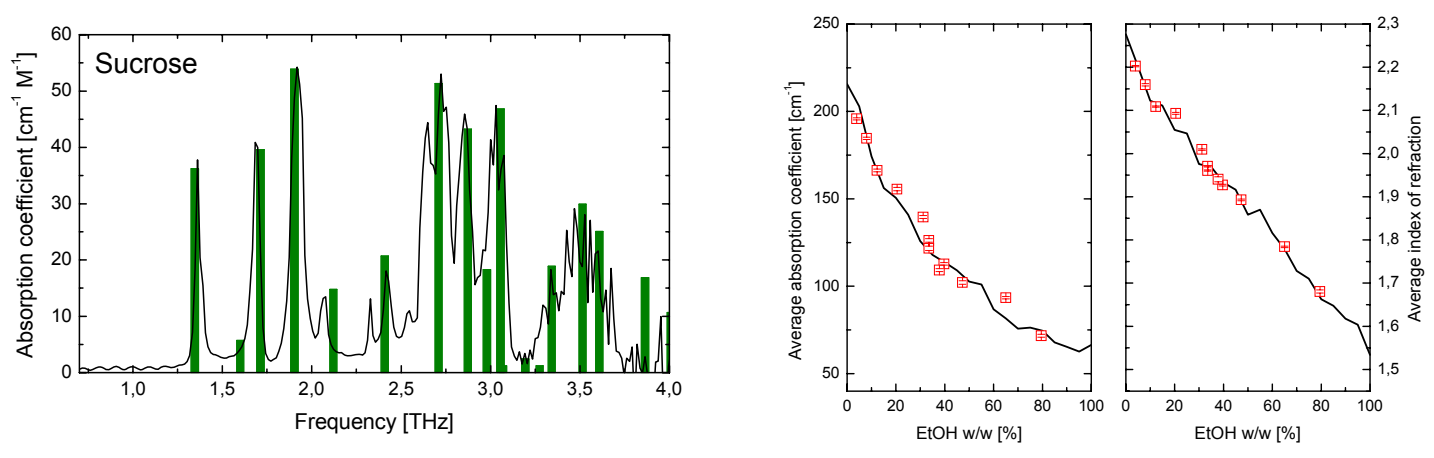

Figure 1: (left panel) Comparison of the measured absorption spectrum (solid curve) and calculated vibrational frequencies of the sucrose crystal. (right panel) Comparison of reference measurements (solid lines) of the average refractive index and absorption coefficient of alcoholwater mixtures with measurements on various beverages with alcohol contents ranging from 5 to $80 \%$ by weight.

The positions as well as the intensities of all the predicted vibrational modes are in very good agreement with the experimentally observed lines. The convincing agreement between experiment and theory allows us to suggest an assignment of the various vibrational frequencies to specific modes of the sucrose crystal.

To illustrate the use of $\mathrm{THz}$ spectroscopy for detection of relevant quantities in non-crystalline systems, Fig.1 (right panel) shows a series of measurements of the absorption coefficient and index of refraction (averaged over the range $0.3-1 \mathrm{THz}$ ) of various beverages with alcohol content ranging between 5 and $80 \%$ by weight (red data points). The solid lines in the plots are the corresponding values of reference mixtures of pure water and pure ethanol.

The good agreement between the properties of the reference mixtures and those of the commercial alcoholic beverages tested here shows that the presence of other ingredients in addition to ethanol and water in the beverage has little influence on the accuracy of the measurement of the alcohol concentration. The accuracy of the measurement is typically in the range of a few percent, currently mainly limited by the size of the reference data set (here recorded in 5\%-intervals) and the temperature stability of the sample.

\section{Conclusion}

The detailed information available with the simulation method presented here about the solid-state $\mathrm{THz}$ vibrational modes of molecules will allow new and important insight into the weak, delocalized forces that hold hydrogen-bonded crystals together. In environments with no long-range order, such as liquids, we have shown that THz spectroscopy still yields important information about the material. Measurements on various food products, such as cheese, butter and meat, indicate that the far infrared properties of such products are very contrary to expectations based on the water content of the sample. This discovery points to that it is not only the amount of water in the sample but also the conditions of the water in the sample that is decisive for the dielectric properties.

[1] M. Walther, B. M. Fischer, and P. Uhd Jepsen, "Noncovalent intermolecular forces in polycrystalline and amorphous saccharides in the far infrared," Chem. Phys. 288, 261-268 (2003)

[2] B. Fischer, M. Hoffmann, H. Helm, G. Modjesch, and P. Uhd Jepsen, "Chemical recognition in terahertz time-domain spectroscopy and imaging", Semiconductor Science and Technology 20, S246-S253 (2005)

[3] M. D. Segall, P. J. D. Lindan, M. J. Probert, C. J. Pickard, P. J. Hasnip, S. J. Clark, and M. C. Payne, "First-principles simulation: ideas, illustrations and the CASTEP code," J. Phys.: Condens. Matter 14, 2717-2744 (2002) 